\title{
Galectin-3: role in ocular allergy and potential as a predictive biomarker
}

\author{
Frans Eberth Costa Andrade, ${ }^{1}$ Mab Pereira Corrêa, ${ }^{2}$ Alexandre Dantas Gimenes, ${ }^{1}$ \\ Myrna Serapião dos Santos, ${ }^{3}$ Mauro Campos, ${ }^{3}$ Roger Chammas, ${ }^{4}$ \\ José Álvaro Pereira Gomes, ${ }^{3}$ Cristiane Damas Gil ${ }^{1,2}$
}

\begin{abstract}
'Departamento de Morfologia e Genética, UNIFESPUniversidade Federal de São Paulo, Sao Paulo, Brazil ${ }^{2}$ Programa de Pós-Graduação em Biociências, Instituto de Biociências, Letras e Ciências Exatas, UNESP-Universidade Estadual Paulista, Sao Jose do Rio Preto, Brazil

${ }^{3}$ Departamento de Oftalmologia e Ciências Visuais, UNIFESPUniversidade Federal de São Paulo, Sao Paulo, Brazil ${ }^{4}$ Departamento de Radiologia e Oncologia, FMUSP_Faculdade de Medicina da Universidade de São Paulo and Instituto do Câncer do Estado de São Paulo, Sao Paulo, Brazil
\end{abstract}

\section{Correspondence to} Dr Cristiane Damas Gil, Departamento de Morfologia e Genética, UNIFESP -Universidade Federal de São Paulo, São Paulo 04023-900, Brazil; cristiane.gil@unifesp.br

Received 17 October 2017 Revised 31 January 2018 Accepted 16 February 2018 Published Online First 3 March 2018

Check for updates

To cite: Andrade FEC, Corrêa MP, Gimenes AD, et al. $\mathrm{Br} J$ Ophthalmol

2018:102:1003-1010.

\section{ABSTRACT}

Aims To evaluate galectin-3 (Gal-3), a $\beta$-galactoside binding protein, as a possible biomarker in ocular allergy and further investigated the role of endogenous Gal-3 in a murine model of ovalbumin (OVA)-induced allergic conjunctivitis (AC).

Methods Conjunctival impression cytology specimens from control and patients with severe vernal

keratoconjunctivitis, treated or untreated, were used to evaluate Gal-3 expression by immunocytochemistry. To investigate the mechanism of action of Gal-3, OVAimmunised BALB/c male wild-type (WT) and Gal-3 null $\left(\mathrm{Gal}-3^{-1}\right)$ mice were challenged with eye drops containing OVA on days 14-16 with a subset of animals pretreated with $0.03 \%$ tacrolimus (TC) or dexamethasone (Dex).

Results Patients with AC and OVA-sensitised WT mice exhibited increased levels of Gal-3 in the conjunctiva compared with control, an effect reverted by the action of Dex and TC therapy. Twenty-four hours after the final OVA challenge, total and anti-OVA lgE levels increased significantly in the blood of OVA-sensitised WT and Gal-3/- mice compared with controls, supporting the efficacy of the AC model. The lack of endogenous Gal-3 exacerbated the local inflammatory response, increasing the influx of eosinophils and mast cell activation. Additionally, OVA-sensitised Gal-3-1- animals exhibited increased $C D 4^{+}$expression in the eyes as well as eotaxin, IL-4, IL-13 and interferon- $\gamma$ levels in the tear fluid compared with WT animals.

Conclusion Gal-3 contributes to the pathogenesis of ocular allergy and represents a relevant therapeutic target.

\section{INTRODUCTION}

Galectins share a highly conserved carbohydrate-recognition domain (CRD) that is responsible for their high affinity for $\beta$-galactosides in glycoconjugates. ${ }^{1}$ In mammals, 15 members of this protein family have been cloned, and based on their structural organisation classified into three subfamilies: proto, chimaera and tandem repeat types. Prototype galectins $(-1,-2,-5,-7,-10$, $-11,-13,-14$ and -15$)$ are composed of a single CRD that is able to dimerise. Galectin-3 (Gal-3) is the only galectin classified as a chimaera-type that consists of one C-terminal CRD linked to an $\mathrm{N}$-terminal domain. The third subfamily consists of tandem-repeat galectins $(-4,-6,-8,-9$ and -12$)$ with two distinct CRDs connected by a flexible peptide linker.
The endogenous expression of Gal-3 protein (also called MAC-2 or L-34) has been observed in several cell types, especially those related to the inflammatory response, such as neutrophils, mast cells, macrophages, lymphocytes and eosinophils. ${ }^{2-5}$ Among the various roles described for Gal-3, the most relevant are those related to the inflammatory response, tumourigenesis, innate and acquired immunity. ${ }^{67}$ In inflammatory processes, Gal-3 has been described as a proinflammatory mediator via the activation of mast cells and basophils, regulation of leucocyte recruitment and monocyte/macrophage cytokine release, chemotaxis and phagocytosis. ${ }^{8-14}$ Despite the proinflammatory roles of Gal-3 in the Th1-driven inflammatory response, its role in allergies, which are Th2-driven, is controversial. In experimental models of ovalbumin (OVA)-induced atopic dermatitis ${ }^{15}$ and asthma, ${ }^{413}$ Gal-3 null mice presented an attenuated inflammatory response characterised by decreased influx of eosinophils, mononuclear cells and lymphocytes in target organs (skin, lung and bronchoalveolar lavage) compared with wild-type (WT) animals. Furthermore, Gal-3 null mice showed a shift in the Th2 response towards Th1 in the asthma model via decrease IL-5 and IL-13 cytokine levels, but not interferon- $\gamma$ (IFN- $\gamma$ ), IL-2 and IL-4. ${ }^{4}$

Conversely, a protective effect of Gal-3 was described in a murine model of OVA-induced asthma using gene therapy. ${ }^{16-18}$ Mice treated with a plasmid encoding Gal-3 (pGal-3) increased Gal-3 protein expression in the lungs and presented a decreased influx of eosinophils and lymphocytes in the bronchoalveolar lavage as well as diminished fibrosis and IL-5 and IL-10 levels compared with non-treated animals. ${ }^{16}{ }^{17}$ Additionally, OVA-challenged mice exhibited higher mRNA expression of so-called suppressor of cytokine signalling (SOCS-1 and SOCS-3) proteins and IL-17 in the lungs than was seen in the pGal-3 mice. ${ }^{18}$ These results suggest that Gal-3 treatment could be a good therapeutic target in allergic diseases.

Given that molecular mechanisms by which Gal-3 modulates cellular responses in allergy are not yet fully determined, we evaluated this protein as a possible biomarker in vernal keratoconjunctivitis (VKC), a chronic allergic inflammation of ocular surface. ${ }^{19}$ Furthermore, we investigated the role of endogenous Gal-3 in a murine model of IgE-mediated allergic conjunctivitis (AC). This study sheds light on the genesis of ocular allergic disorders and may lead to new therapies for the treatment of these diseases. 
Table 1 Patient characteristics by group

\begin{tabular}{lll}
\hline & Groups \\
\cline { 2 - 3 } & AC & Control \\
\hline Number of subjects & 29 & 9 \\
Sex (male:female) & $23: 6$ & $4: 5$ \\
\hline Age (years) (mean \pm SD) & $13.31 \pm 3.3$ & $27.55 \pm 9.48$ \\
\hline Local treatment & \multicolumn{2}{l}{ Number of subjects } \\
\hline Antiallergic drugs* only & 9 & 0 \\
\hline Antiallergic drugs and corticosteroidt & 2 & 0 \\
\hline Antiallergic drugs and tacrolimus & 9 & 0 \\
\hline Antiallergic drugs, corticosteroid and tacrolimus & 6 & 0 \\
\hline Untreated & 3 & 9 \\
\hline
\end{tabular}

${ }^{*}$ Antiallergic drugs consist of antihistamine and mast cell stabiliser.

tCorticosteroids consist of dexamethasone or prednisolone.

$A C$, allergic conjunctivitis.

\section{MATERIALS AND METHODS}

\section{Patients}

The study was conducted in compliance with recognised international standards and the principles of the Declaration of Helsinki. Informed consent about the procedures was obtained. This study was carried out from August 2015 to May 2016 at the Cornea and External Diseases Service of the Department of Ophthalmology of the Federal University of São Paulo (UNIFESP), Brazil.

The cohort comprised 29 patients with severe VKC (AC group) diagnosed by a detailed history and characteristic clinical signs, such as itching, foreign body sensation, photophobia and tearing, together with typical findings of limbal inflammatory activity (ie, limbal hyperaemia and Horner-Trantas dotseosinophilic concretions) and giant papillae on the upper tarsal conjunctiva and epithelial keratitis. ${ }^{20} 21$ Conjunctival epithelial cells were sampled from the more severely affected eye defined by a clinical evaluation of signs and symptoms of ocular allergy. ${ }^{20}$ Nine healthy volunteers with no history of ocular or systemic allergic disease or history of drug or contact lens use were recruited from the community of the UNIFESP to the study as a control group. Demographic data and previous treatment histories of the enrolled patients at the time of cell collection are shown in table 1.

\section{Conjunctival impression cytology and immunocytochemistry}

Impression cytology was performed as previously described. ${ }^{22}$ After application of topical anaesthesia (proparacaine $0.5 \%$ ) into the eye, four strips of $5 \times 7 \mathrm{~mm}$ pore filter papers $(45 \mu \mathrm{m}$; Millipore) were gently placed over the bulbar conjunctiva for $2-5 \mathrm{~s}$. The filter paper was then removed and immersed in phosphate-buffered $4 \%$ paraformaldehyde for 24 hours at $4{ }^{\circ} \mathrm{C}$. After washing, samples were fixed on glass slides and subjected to cellulose digestion using viscozyme (Sigma-Aldrich, Michigan, USA) for 3 hours at $37^{\circ} \mathrm{C}$. The endogenous peroxidase activity was blocked and the cells were incubated overnight at $4^{\circ} \mathrm{C}$ with a rabbit polyclonal anti-Gal-3 antibody (Santa Cruz Biotechnology, California, USA), diluted 1:200 in phosphate buffer saline (PBS) containing 1\% bovine serum albumin (BSA). Some samples were incubated with $1 \%$ BSA in PBS instead of the primary antibody as a negative control for the reaction. After washing, cells were incubated with a biotinylated secondary antibody (LAB-SA Detection kit, Invitrogen, Paisley, UK). Positive staining was detected using a peroxidase-conjugated streptavidin complex and colour was developed using DAB substrate (Invitrogen). Densitometry analysis of Gal-3 immunostaining was performed in the epithelial cells ( $\mathrm{n}=60$ cells/patient). The values were obtained as arbitrary units (a.u.) between 0 and 255 using AxioVision software on an Axioskop 2 mot plus Zeiss microscope (Carl Zeiss, Jena, Germany). The data are expressed as the mean \pm SEM of a. $u$.

\section{Animals}

Male WT and Gal-3 deficient (Gal-3 $3^{-/-}$) BALB/c mice, weighing 20-25 g, were randomly distributed into six groups $(n=6 /$ group). Gal- $3^{-/-}$mice were generated as described ${ }^{23}$ and backcrossed to BALB/c mice for nine generations. The animals were housed in a 12 hours light-dark cycle and were allowed food and water ad libitum. All experimental procedures were submitted and approved by the Ethics Committee in Animal Experimentation of the Federal University of São Paulo-UNIFESP (CEUA $\left.n^{\circ} 5448030215\right)$.

\section{Allergic conjunctivitis model and treatment protocols}

$\mathrm{BALB} / \mathrm{c}$ mice were immunised on days 0 and 7 with a subcutaneous injection of $5 \mu \mathrm{g}$ of ovalbumin (OVA, grade V) and $15 \mathrm{mg}$ / $\mathrm{mL}$ of aluminium hydroxide adjuvant diluted in $200 \mu \mathrm{L}$ of sterile saline as reported previously. ${ }^{24}{ }^{25}$ On days 14,15 and 16 after an intraperitoneal (i.p.) injection of anaesthesia with ketamine $(100 \mathrm{mg} / \mathrm{kg})$ and xylazine $(20 \mathrm{mg} / \mathrm{kg})$, mice received direct application of $250 \mu \mathrm{g}$ of OVA in $10 \mu \mathrm{L}$ of sterile saline onto the conjunctival sac. Control animals received sterile saline alone.

To determine the effect of tacrolimus (TC) or dexamethasone (Dex) administration on Gal-3 expression, OVA-sensitised mice were pretreated on days 14, 15 and 16 with topical TC at $0.03 \%$ (Ophthalmos, São Paulo, Brazil) or Dex (1 mg/kg, Sigma-Aldrich) i.p., $15 \mathrm{~min}$ before the application of OVA. ${ }^{24-26}$

Twenty-fourhours after the last OVA challenge, mice were anaesthetised and blood obtained via cardiac puncture using a syringe with 10\% EDTA for the analysis of IgE levels and leucocyte quantification. The lacrimal fluid was collected by direct instillation of $20 \mu \mathrm{l}$ of sterile saline into the conjunctival sac of each eye, providing a pool of $40 \mu \mathrm{L} /$ animal. The animals were then euthanised and the eyes, eyelids, spleen and peritoneal washed and collected.

\section{Analysis of total IgE, IgE anti-ovalbumin and Gal-3 levels}

Blood from various experimental conditions was centrifuged at $600 \mathrm{x} g$ for $10 \mathrm{~min}$ to collect the plasma and determine the total IgE and anti-OVA levels by ELISA. The concentration of total IgE and anti-OVA were measured using commercially available mouse total IgE and anti-OVA immunoassay kits (BioLegend, San Diego, California, USA and Cayman Chemical, Ann Arbour, Michigan, USA, respectively), in accordance with the manufacturer's guidelines. Gal-3 levels in plasma and spleen homogenates were determined using a commercially available kit (RayBiotech, Georgia, USA), in accordance with the manufacturer's guidelines. All experiments were conducted in duplicate and the data expressed as the mean \pm SEM protein $(\mathrm{ng} / \mathrm{mL}$ or $\mathrm{pg} / \mathrm{mL})$.

\section{Ultrastructural immunocytochemical analysis}

To detect the localisation of endogenous Gal-3 and phosphorylated extracellular signal-regulated kinase (pERK) in inflammatory cells, peritoneal wash was collected and centrifuged at 600 $\mathrm{x} g$ for $15 \mathrm{~min}$ and cells were resuspended and fixed in 4\% paraformaldehyde, $0.5 \%$ glutaraldehyde, $0.1 \%$ sodium cacodylate buffer ( $\mathrm{pH} 7.4$ ) for 24 hours at $4^{\circ} \mathrm{C}$. Peritoneal cells were washed in sodium cacodylate, dehydrated through a graded series of methanol washes and embedded in LR Gold (Sigma-Aldrich, St. 
Louis, Missouri, USA). Ultrathin sections ( $90 \mathrm{~nm})$ of peritoneal cells were placed on nickel grids and incubated sequentially with the following reagents at room temperature: (1) $0.1 \mathrm{~mol} / \mathrm{L}$ PBS containing 1\% egg albumin (PBEA); (2) PBEA 5\% for $30 \mathrm{~min}$; (3) rabbit polyclonal anti-Gal-3 antibody or anti-pERK (1:50 in PBEA 1\%; Santa Cruz Biotechnology) for 2 hours and (4) three washes (5 min each) in 1\% PBEA. To detect Gal-3 or pERK, a goat anti-rabbit IgG antibody (1:50 in PBEA) conjugated to $15 \mathrm{~nm}$ colloidal gold (British Biocell, Cardiff, UK) was added. After 1 hour, the sections were washed extensively in $1 \%$ PBEA and then in distilled water. These sections were stained with uranyl acetate and lead citrate and examined using a ZEISS EM900 electron microscope.

\section{Histopathological analysis and quantification of inflammatory cells in palpebral conjunctiva}

Eyelids were fixed in $4 \%$ paraformaldehyde for 24 hours, washed in tap water, dehydrated in a increasing ethanol series and embedded in paraffin. Sections of $3 \mu \mathrm{m}$ were obtained using a Leica RM2155 microtome (Leica Microsystems, Nussloch, Germany) and subsequently stained with haematoxylin-eosin or $0.5 \%$ toluidine blue for histopathology and inflammatory cell quantification. Eosinophils and mast cells were quantified using a $40 \times$ objective on an Axio Scope A1 Zeiss microscope (Carl Zeiss). Three semiserial sections of palpebral conjunctiva were analysed per animal and the area was determined using AxioVision software (Carl Zeiss). Values are expressed as the mean \pm SEM of cells per $\mathrm{mm} .^{2}$

\section{Immunohistochemistry}

The detection of Gal-3 in the palpebral conjunctiva was performed using a primary rabbit polyclonal anti-Gal-3 antibody (1:200 in PBS with 1\% BSA) (Santa Cruz Biotechnology) and a secondary biotinylated antibody (LAB-SA Detection kit, Invitrogen, Paisley, UK). Positive staining was detected using a peroxidase-conjugated streptavidin complex, and colour was developed using DAB substrate (Invitrogen). The sections were counterstained with haematoxylin. Densitometric analyses for Gal-3 immunostaining were performed in the epithelium and lamina propria (LP) of palpebral conjunctiva ( $\mathrm{n}=6$ animals/group). The values were obtained as a.u. between 0 and 255 using AxioVision software on an Axioskop 2 mot plus Zeiss microscope (Carl Zeiss). The data are expressed as the mean \pm SEM of a. u.

\section{Western blot analysis}

Eyes were sonicated in a $50 \mathrm{mM}$ Tris- $\mathrm{HCl}, 150 \mathrm{mM} \mathrm{NaCl}$ and $1 \%$ Triton-X, pH 7.4 buffer containing a complete protease inhibitor cocktail (Roche Applied Science, Mannheim, Germany). Subsequently, samples were centrifuged at $10000 \mathrm{xg}$ for $20 \mathrm{~min}$ at $4^{\circ} \mathrm{C}$ to obtain organ supernatants. Protein levels were determined by Bradford assay and normalised prior to boiling in Laemmli buffer (Bio-Rad Laboratories, USA). Protein extracts $(30 \mu \mathrm{g}$ per lane) of eye from the indicated experimental conditions were loaded onto a $12 \%$ sodium dodecyl sulfate-polyacrylamide gel for electrophoresis together with appropriate molecular weight markers (Bio-Rad Life Science, USA) and transferred to ECL Hybond nitrocellulose membranes. Reversible protein staining of the membranes with $0.1 \%$ Ponceau-S in $5 \%$ acetic acid (Santa Cruz Biotechnology) was used to verify protein transfer. Membranes were incubated for $15 \mathrm{~min}$ in 5\% BSA in Tris-buffered saline (TBS) prior to incubation with antibodies. Primary antibodies used herein: goat polyclonal anti-mouse mast cell protease 6 (mMCP6; 1:5000) (R\&D Systems, Minneapolis, USA), rat monoclonal anti-CD4 (1:500) (Imuny-Rheabiotech, Campinas, Brazil), rabbit anti-Gal-3 (1:200) (Santa Cruz Biotechnology) and anti-GAPDH (glyceraldehyde-3-phosphate dehydrogenase; 1:5000) (Sigma-Aldrich), all diluted in TBS with $0.1 \%$ Tween 20. Post primary antibody incubation, membranes were washed for $15 \mathrm{~min}$ with TBS and subsequently incubated for $60 \mathrm{~min}$ at room temperature with the appropriate secondary antibodies. Secondary antibodies were peroxidase-conjugated rabbit antigoat, goat anti-rabbit or anti-rat IgG (1:2000) (Thermo Fisher Scientific, Michigan, USA). Finally, membranes were washed for 15 min with TBS, and immunoreactive proteins were detected (Westar Nova 2.0 chemiluminescent substrate kit; Cyanagen, Bologna, Italy) using a GeneGnome5 chemiluminescence detection system (SynGene, Cambridge, UK).

\section{Analysis of cytokines and chemokines in tear fluid}

Multiplex analysis was performed with $25 \mu \mathrm{L}$ of the tear fluid using the MILLIPLEX MAP mouse cytokine/chemokine panel (MT17MAG47K-PX25; Millipore, USA) and MAGPIX Multiplexing Instrument (Millipore) according to the manufacturer's instructions. Four analytes were measured: IL-4, IL-13, IFN- $\gamma$ and eotaxin. The concentration of analytes was determined by MAGPIX Xponent software (Millipore, Massachusetts, USA), and the results are reported as the mean \pm SEM of analytes $(\mathrm{pg} / \mathrm{mL})$.

\section{Statistical analyses}

The data were analysed using GraphPad Prism V.5.0 software. Results were confirmed to follow a normal distribution using Kolmogorov-Smirnov test of normality with Dallal-WilkinsonLillie for corrected $\mathrm{p}$ value. Data that passed the normality assumption were analysed using analysis of variance with Bonferroni posthoc test (multiple group comparison) or $t$ test (two independent groups). Data that failed the normality assumption were analysed using the non-parametric Kruskal-Wallis followed by Dunn post-test (multiple group comparison) or Mann-Whitney test (two independent groups). Differences were considered statistically significant at a value of $p<0.05$.

\section{RESULTS}

\section{Keratoconjunctivitis is associated with increased Gal-3 expression in the bulbar conjunctival epithelium}

A total of 38 patients were recruited to this study and distributed in the followed groups: control ( $\mathrm{n}=9$ patients/group), untreated keratoconjunctivitis (AC; $\mathrm{n}=3$ ), treated with antiallergic drugs only $(\mathrm{AH} ; \mathrm{n}=9)$ or antiallergic drugs and corticosteroid $(\mathrm{AH}+\mathrm{CORT}$, $\mathrm{n}=2$ ), tacrolimus ( $\mathrm{AH}+\mathrm{TC} ; \mathrm{n}=9$ ) or tacrolimus and corticosteroid $(\mathrm{AH}+\mathrm{TC}+\mathrm{CORT} ; \mathrm{n}=6) . \mathrm{Gal}-3$ protein expression was detected in the nucleus and cytoplasm of bulbar conjunctival epithelial cells from all study groups. Strong Gal-3 immunoreactivity was observed in the nucleus and cytoplasm of the epithelium in the untreated AC group compared with the control and $\mathrm{AH}+\mathrm{TC}+\mathrm{CORT}$ therapy (figure $1 \mathrm{~A}-\mathrm{C}$ ). No immunostaining was detected in the sample used as a negative control (figure 1D). Densitometry confirmed the histological observations, showing a significant increase of Gal-3 expression in the both subcellular compartments (nucleus and cytoplasm) of the epithelium in the AC group in relation to the control (figure $1 \mathrm{E}$ and $\mathrm{F}$ ). $\mathrm{AH}$ and $\mathrm{AH}+\mathrm{TC}$ therapies also maintained high nuclear and cytoplasmic Gal-3 levels in the epithelium compared with the control group. Conversely, corticosteroid treatments $(\mathrm{AH}+\mathrm{CORT}$ and $\mathrm{AH}+\mathrm{TC}+\mathrm{CORT})$ significantly decreased Gal-3 expression in the two compartments analysed in comparison to the AC group. 

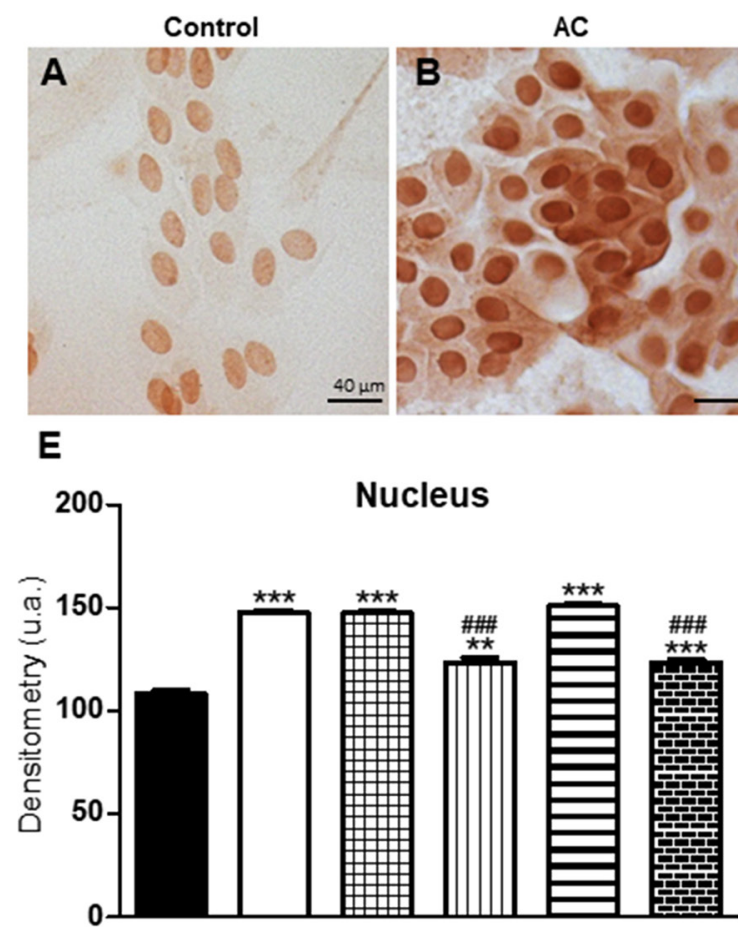

AC

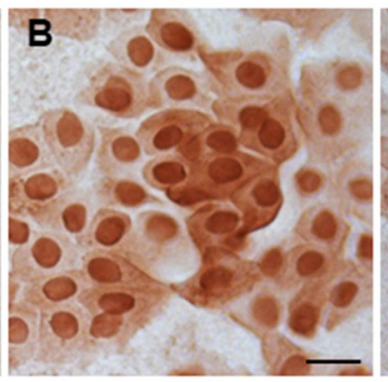

$F$
$\mathrm{AH}+\mathrm{TC}+\mathrm{CORT}$

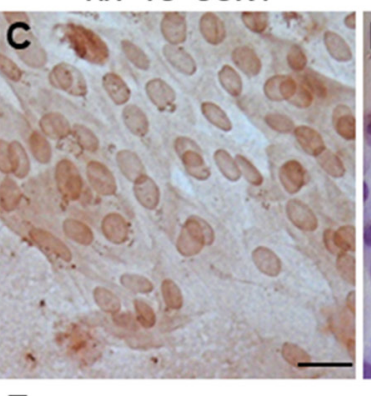

F 200

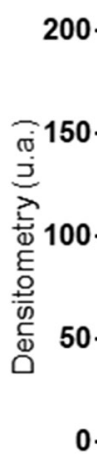

Negative Control

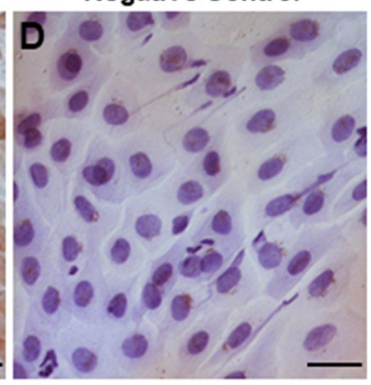

- Control

口 AC

曲 $\mathrm{AH}$

血 AH+CORT

向 $\mathrm{AH}+\mathrm{TC}$

AH+TC+CORT
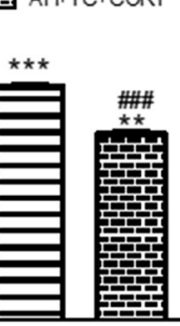

Figure 1 Gal-3 expression in human conjunctival epithelium using impression cytology. (A-C) Epithelial cells from AC group displayed intense Gal-3 immunoreactivity in the nucleus and cytoplasm compared with the control and treated groups (AH+TC+CORT). (D) Absence of immunoreactivity in the negative control samples. Counterstain: Haematoxylin. Bars: $20 \mu \mathrm{m}$. Densitometric analysis of Gal-3 expression in the nucleus (E) and cytoplasm (F) of conjunctival epithelial cells. Data (arbitrary units, a.u.) represent the mean \pm SEM of Gal-3 immunoreactivity. ${ }^{*} P<0.05,{ }^{* *} P<0.01$; ${ }^{* *} P<0.001$ vs Control; ${ }^{\# \#} \mathrm{P}<0.001$ vs $A C$ (Kruskal-Wallis, Dunn post-test). AC, untreated keratoconjunctivitis; $A H$, antihistamine; $A H+C O R T$, antihistamine and corticosteroid; $\mathrm{AH}+\mathrm{TC}$, antihistamine and tacrolimus; $\mathrm{AH}+\mathrm{TC}+\mathrm{CORT}$, antihistamine, tacrolimus and corticosteroids.

\section{Gal-3 protein expression is also increased in murine experimental AC}

We performed immunohistochemistry studies to ascertain whether Gal-3 expression was modulated in the palpebral conjunctiva under AC and pharmacological treatments. Gal-3 expression was detected in the surface epithelial cells and LP in the conjunctiva of the control group (figure 2A). After 24 hours post-OVA challenge, a marked increase in Gal-3 immunoreactivity was demonstrated for both tissues in relation to control, effect abrogated by TC and Dex treatments (figure 2A-D). No immunostaining was detected in the negative control samples (figure 2E). The histological findings were supported by densitometry analyses of Gal-3 expression in the epithelial cells and LP of the conjunctiva (figure $2 \mathrm{~F}$ and $\mathrm{G}$ ). Despite modulation of local Gal-3 levels, we did not detect changes in spleen and plasma Gal-3 levels under different experimental conditions (figure $2 \mathrm{H}$ and $\mathrm{I}$ ).

Ultrastructural studies of peritoneal cells using postembedding immunogold labelling showed nuclear and cytoplasmic localization of Gal-3 in the mast cells, lymphocytes and eosinophils (figure 2J-L). In mast cells and eosinophils, gold particles associated with Gal-3 were mainly detected within cytoplasmic granules and, to a lesser extent, throughout the cytosol, suggesting that both cell types are a potential source of this lectin.

\section{Local allergic inflammatory response is exacerbated in Gal-3- tanimals}

Once we had determined that human and murine AC increased endogenous Gal-3 expression, the next step in our study was to evaluate the role of this protein in the inflammatory response induced by AC model. For this purpose, OVA-induced AC was performed in WT and Gal-3 ${ }^{-/}$BALB/c mice.

The absence of endogenous Gal-3 was confirmed by Western Blot using the eye homogenates. As expected, pooled extracts of WT eyes ( $n=3$ animals per group) from SHAM and AC groups demonstrated a corresponding band for Gal-3 $(\sim 29 \mathrm{kDa})$ while in Gal- $3^{-/}$animals, no band was detectable (figure 3A). After 24 hours of the last OVA challenge, total and anti-OVA IgE levels were significantly increased in the plasma of WT and Gal-3 $3^{-1-}$ animals from the AC group compared with the respective SHAM controls (figure $3 \mathrm{~B}$ and $\mathrm{C}$ ).

Histological analyses of the palpebral conjunctivae in WT and Gal- $3^{-/}$control groups showed a normal appearance, with stratified squamous epithelium and LP (figure 4A and C). OVA-sensitised mice exhibited an intense influx of eosinophils into the LP that was exacerbated in the Gal- $3^{-/}$mice (figure $4 \mathrm{~A}-\mathrm{D}$ and I). Conjunctival sections stained with toluidine blue revealed the presence of mast cells with metachromatic cytoplasmic granules in the LP (figure 4E-H). AC groups (WT and Gal- $3^{-/}$) displayed both intact and degranulated cells (figure 4F and $\mathrm{H}$ ), which were markedly increased in the Gal-3/- mice (figure $4 \mathrm{~J}$ and $\mathrm{K})$. These morphological findings were corroborated by the analysis of mMCP6 levels in AC eye homogenates that showed increased immunoreactivity in the Gal- $3^{-/}$group compared with WT (figure 4L). The AC-induced inflammatory response also included local lymphocyte recruitment, as demonstrated by immunoreactive bands for $\mathrm{CD}^{+}$in both genotypes, with a significant increase in the Gal- $3^{-/}$eye samples (figure $5 \mathrm{~A}$ ). In addition, pERK was significantly increased in the cytoplasm and nucleus of Gal-3 $3^{-/}$peritoneal lymphocytes from AC group compared with WT (figure 5B), as revealed by ultrastructural 

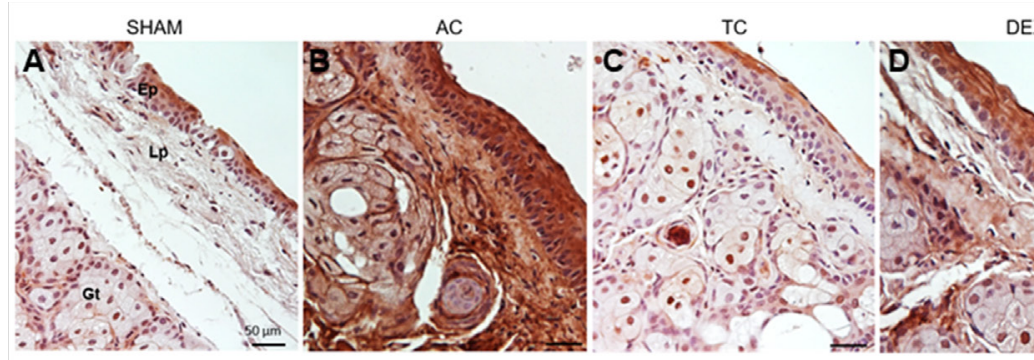

DEX
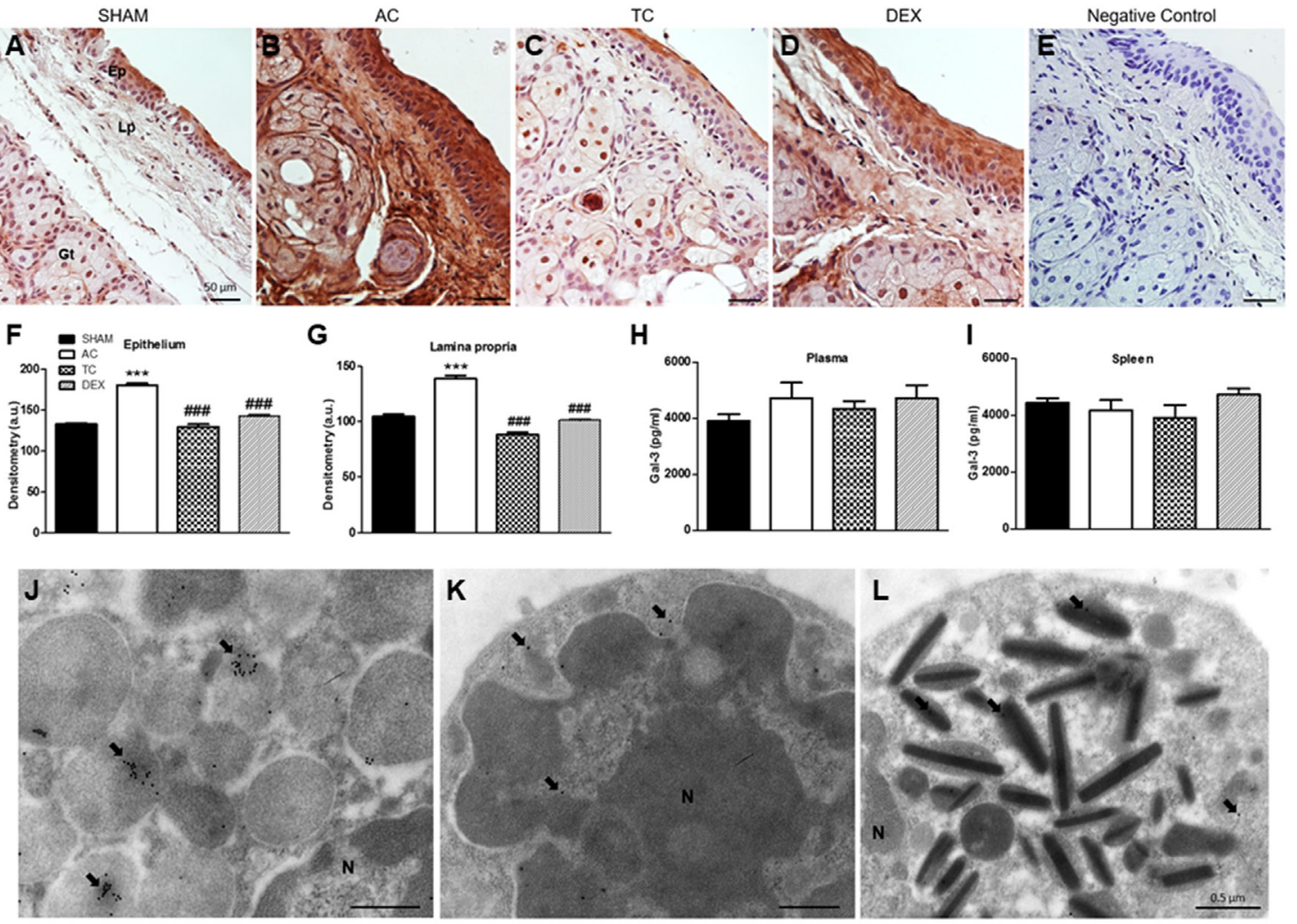

Figure 2 Gal-3 expression in the AC mouse model. (A-D) AC induced strong Gal-3 immunoreactivity in the LP and Ep of palpebral conjunctivae in relation to control, TC-treated and Dex-treated groups. (E) No immunostaining was detected in the negative control sample. Counterstain: Haematoxylin. Bars: $50 \mu \mathrm{m}$. (F,G) Densitometric analysis of Gal-3 expression in the epithelium and LP. Data (arbitrary units, a.u.) represent the

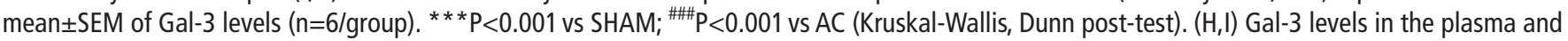
spleen homogenates. Data displayed as mean \pm SEM of Gal-3 levels $(\mathrm{pg} / \mathrm{mL}$ ) ( $\mathrm{n}=6$ animals/group). (J-L) Ultrastructural localisation of Gal-3 in mast cell (J), lymphocyte (K) and eosinophil (L). Gal-3 immunogold particles (arrows) were detected in the nucleus, cytosol and cytoplasmic granules. SHAM, control group. AC, allergic conjunctivitis; DEX, treated with dexamethasone; Ep, epithelium; LP, lamina propria; TC, treated with tacrolimus.

studies. The pronounced local inflammation demonstrated in $\mathrm{Gal}^{-3^{--}}$animals was corroborated by the analysis of tear fluid. Data revealed that Gal- $3^{-/}$mice undergoing AC had significantly increased levels of eotaxin, IL-4, IL-13 and IFN- $\gamma$ compared with WT animals (figure 5C).

\section{DISCUSSION}

Given the pathophysiology of ocular allergy continues to be a challenging task for ophthalmologists and the fact that current therapies increased risk of adverse effects, ${ }^{27}$ it is pertinent to investigate new therapeutic targets that might lead to the development of anti-inflammatory agents.
Current knowledge about the role of Gal-3 in allergic inflammation is still controversial with some studies pointing to a proinflammatory profile ${ }^{43}$ and others to an anti-inflammatory role. ${ }^{16-18}$ Thus, with this study, we broaden the knowledge of Gal-3 in the regulation of the inflammatory response to ocular allergy using cytology, histological, immunohistochemistry and biochemical analyses.

Considering Gal-3 as a possible biomarker of ocular allergy, we initially investigated its expression in the conjunctival epithelium by impression cytology of healthy controls and patients with VKC, with or without pharmacological treatment. Impression cytology is a non-invasive method for evaluating the ocular
A

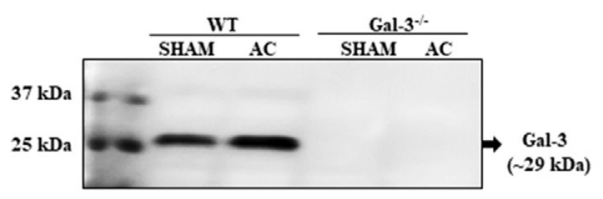

B

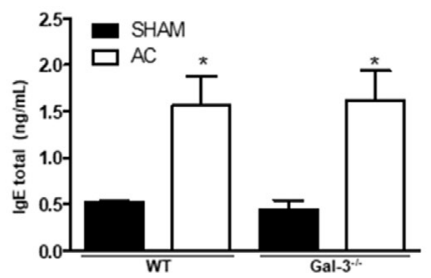

C

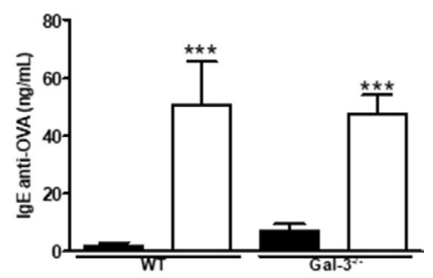

Figure 3 Gal-3, total and anti-OVA IgE levels. (A) Immunoblot analysis confirmed the absence of Gal-3 expression in the Gal-3 ${ }^{-1-}$ groups (SHAM and AC) whereas immunoreactive bands $(\sim 29 \mathrm{kDa})$ were detected in the pooled extracts of WT mouse eyes $(\mathrm{n}=3$ animals/group). The data illustrate one representation of two independent experiments. $(\mathrm{B}, \mathrm{C})$ Total and anti-OVA IgE levels. Data represent mean $\pm \mathrm{SEM}$ of $\mathrm{IgE}(\mathrm{ng} / \mathrm{mL})(\mathrm{n}=6 \mathrm{animals} / \mathrm{group})$. ${ }^{*} \mathrm{P}<0.05,{ }^{* *} \mathrm{P}<0.001$ vs SHAM of respective genotype (t-test). AC, allergic conjunctivitis; OVA, ovalbumin; SHAM, control group; WT, wild type. 


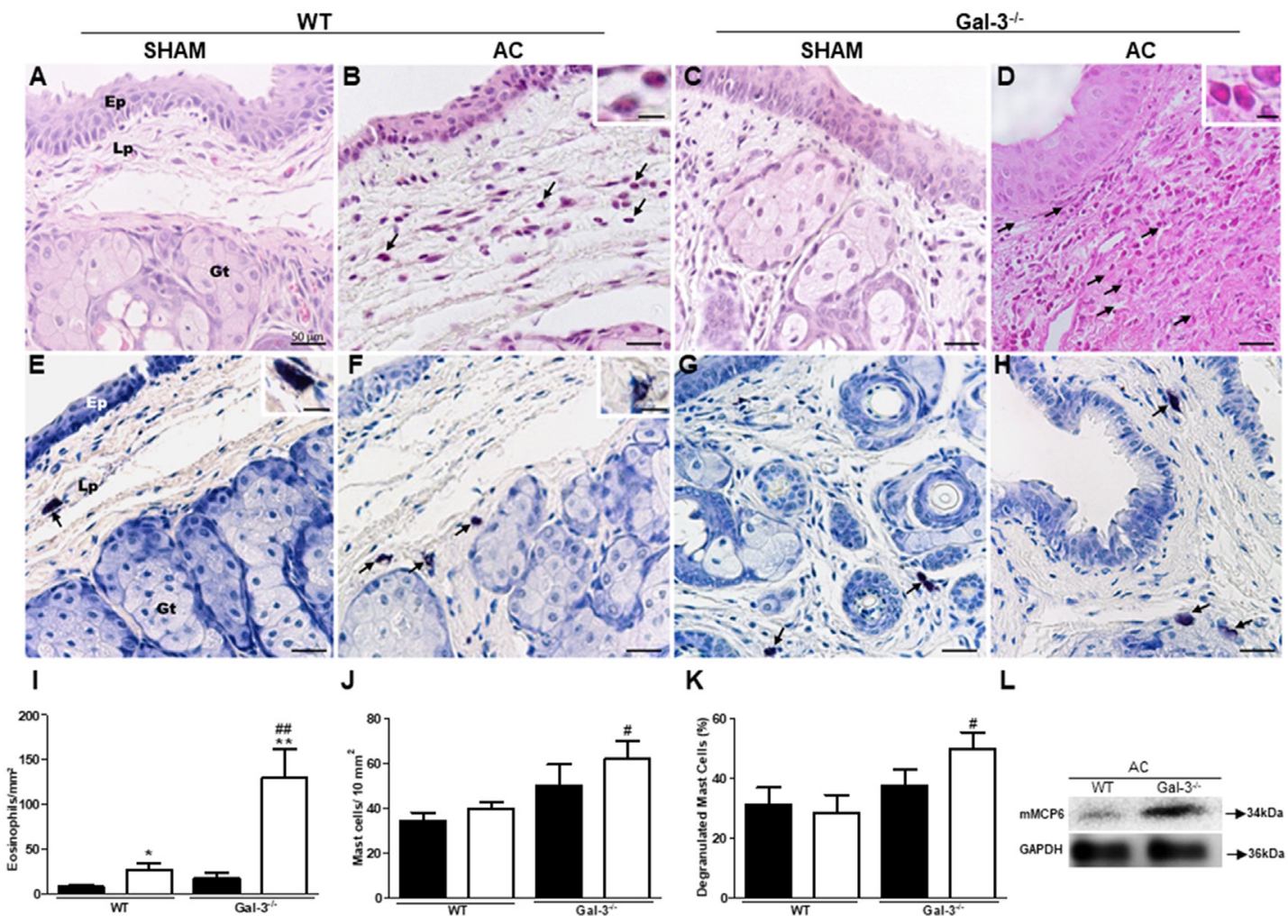

Figure 4 Effect of lack of endogenous Gal-3 in the local inflammatory response in $A C$ model. (A,C,E,G) Histological view of conjunctival membranes from WT and Gal-3 $3^{-1-}$ SHAM groups showing normal morphology of stratified squamous Ep, LP and Gt. (E,G) Intact mast cells (arrows; inset) with metachromatic cytoplasmic granules. (B,D,F,H) Intense influx of eosinophils and mast cells (arrows) in the LP from WT and Gal- $3^{-1-} A C$ groups. Insets: detail of eosinophils (B,D) and degranulated mast cell (F). Stain: haematoxylin-eosin (A-D); toluidine blue (E-H). Bars: $20 \mu \mathrm{m} ; 10 \mu \mathrm{m}$ (insets). (I-K) Quantification of eosinophils and mast cells. Data represent mean \pm SEM of the number of cells $/ \mathrm{mm}^{2}$ and proportion of degranulated mast cells percentage ( $n=6 /$ group). ${ }^{*} \mathrm{P}<0.05$; ${ }^{*} \mathrm{P}<0.01$ vs SHAM of respective genotype; ${ }^{\#} \mathrm{P}<0.05$; ${ }^{\# \#} \mathrm{P}<0.01$ vs WT AC (ANOVA, Bonferroni post-test). Western blot analysis to measure mMCP6 levels in the pooled extracts of eyes ( $n=3$ animals/group) from mice AC WT or AC Gal- $3^{-1}$. GAPDH was used as a protein loading control (data represent one illustrative blot from two independent experiments). AC, allergic conjunctivitis; ANOVA, analysis of variance; Ep, epithelium; Gt, tarsal gland; LP, lamina propria; $\mathrm{mMCP6}$, mouse mast cell protease 6; OVA, ovalbumin; SHAM, control group; WT, wild type.

surface, which includes the epithelium of the conjunctiva and cornea. It is an important tool in ophthalmology, helping to develop a more-accurate diagnosis in several surface ocular diseases, in addition to guaranteeing better quality of the samples in relation to the cytology obtained with ocular surface scraping. ${ }^{28} 29$

The immunocytochemistry showed a significant increase in Gal-3 expression in the conjunctival epithelium with VKC in relation to the control patients. Despite VKC pathogenesis is much more complex than a mere type 1 hypersensitivity reaction, ${ }^{19}$ similar results were observed in the murine conjunctival membrane under OVA-induced conjunctivitis. In fact, increased expression of Gal-3 protein has also been reported in some experimental models of allergy. In a model of allergic rhinitis in mice, high levels of Gal-3 mRNA were detected in the nasal mucosa of animals challenged with OVA. ${ }^{30}$ High Gal-3 expression in bronchoalveolar lavage and lung of mice was also demonstrated in OVA-induced asthma, specifically located in the alveolar macrophages. ${ }^{4}{ }^{13} 31$ In these studies, Gal-3 expression was associated with mobilisation of eosinophils during allergic inflammation. Moreover, increased Gal-3 expression was detected in the skin of patients and mice with atopic dermatitis, particularly localised in the lymphocytes of the inflammatory infiltrate. ${ }^{15}$

Among the different pharmacological treatments for patients with VKC, corticosteroids were identified as an effective immunoregulatory drug affecting Gal-3 expression. These findings were corroborated by the experimental AC model that demonstrated a decrease in the Gal-3 levels in the mouse conjunctiva after Dex administration. A similar effect was exhibited for TC treatment. Indeed, the endogenous expression of Gal-3 protein and its mRNA in monocytes/macrophages is regulated by glucocorticoids (Dex and hydrocortisone) and non-steroidal anti-inflammatory drugs (indomethacin and aspirin). ${ }^{5}$ However, to the best of our knowledge, there are no reports on the effect of TC on this lectin. Thus, for the first time we demonstrate the negative regulatory effect of immunosuppressive TC on Gal-3 expression under $\mathrm{AC}$, which was not observed in the human conjunctival epithelial cells from patients treated with TC in combination with antihistamine. Downregulation of Gal-3 levels induced by corticosteroids and immunosuppressive drugs is probably related to the diminished influx of inflammatory cells (mast cells and eosinophils) to the conjunctival membrane. These cells represent an important source of this lectin for the tissue.

To further understand this relationship of endogenous Gal-3 in association with the ocular allergic inflammatory response, our next step was to investigate the role of this protein in AC using WT and Gal- $3^{-1}$ animals. The efficacy of the AC model was validated by the increase of the total and anti-OVA IgE plasma levels in the WT and Gal- $3^{-1-}$ animals compared with their respective controls. In addition, the allergic inflammatory response in WT 

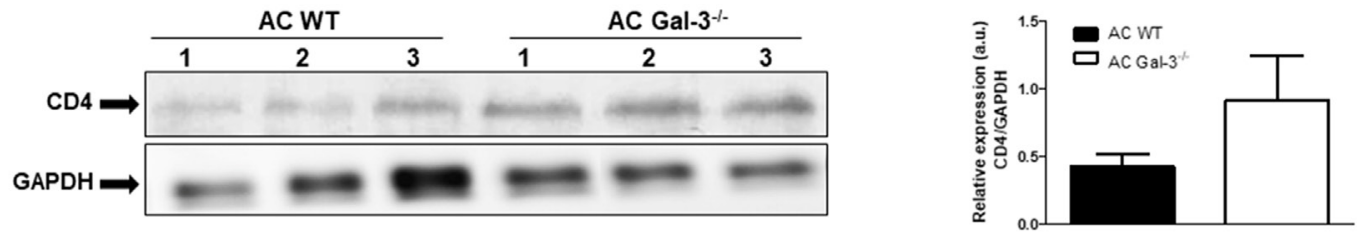

B
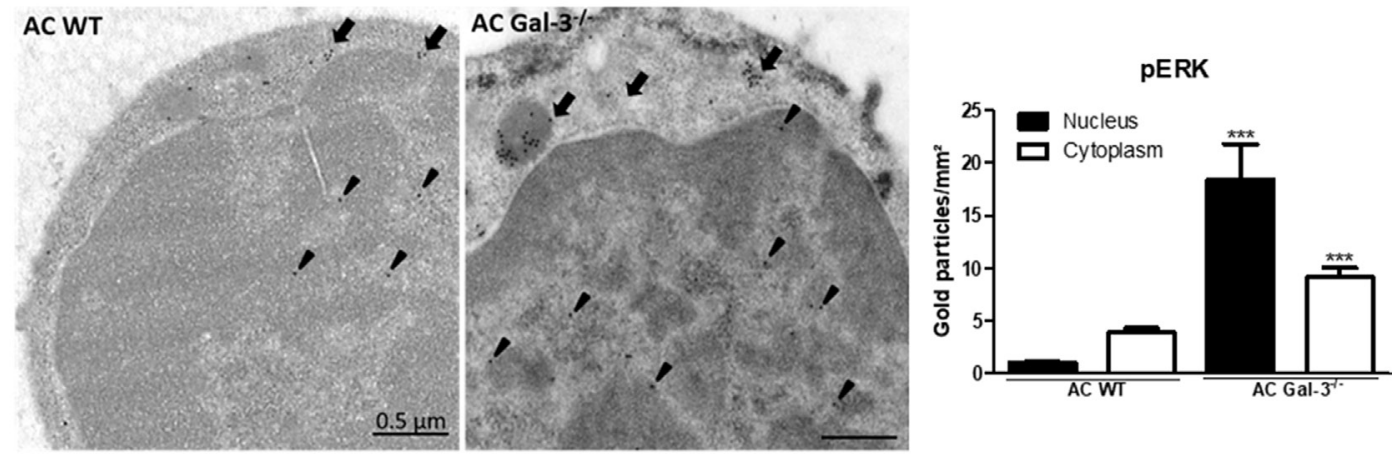

\section{C}
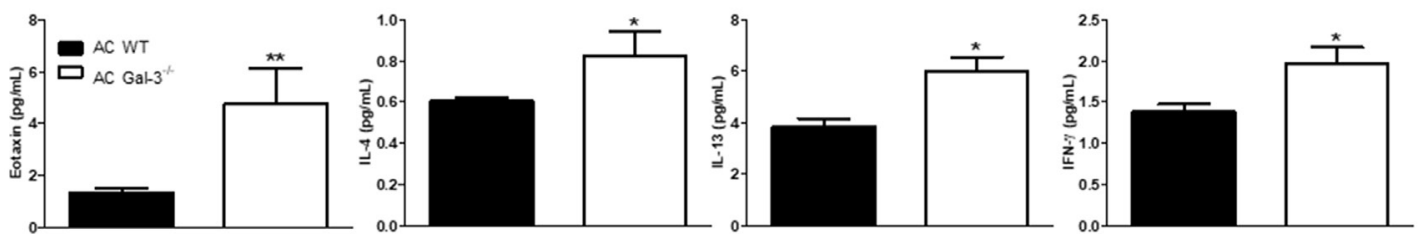

Figure 5 Effect of lack of endogenous Gal-3 in the immune response in AC model. (A) CD4 expression in eye homogenates from WT and Gal- $3^{-1-}$ AC mice $(\mathrm{n}=3 /$ group, lanes $1-3)$. Gal- $3^{-1 /}$ mice presented intense immunoreactive bands for CD4 ( 51 kDa) in relation to WT. GAPDH was used as a protein loading control. Immunoreactive bands were semiquantified by densitometry and are expressed as arbitrary units (a.u.) of the ratio of CD4/GAPDH. (B) Ultrastructural localisation of pERK in peritoneal lymphocytes. Gal-3-1- cells exhibit increased cytoplasmic (arrows) and nuclear (arrowheads) pERK expression compared with WT cells. Data are the mean \pm SEM of immunogold particles per $\mu \mathrm{m}^{2^{* * *}} \mathrm{P}<0.001 \mathrm{vs} \mathrm{WT} \mathrm{AC} \mathrm{(} t$ test). (C) Levels of eotaxin, IL4, IL-13 and IFN- $\gamma$ in tear fluid. Values are expressed as the mean \pm SEM of the chemokine/cytokines ( $\mathrm{pg} / \mathrm{ml} ; \mathrm{n}=6 / \mathrm{group})$. ${ }^{*} \mathrm{P}<0.05$ and ${ }^{*}{ }^{*} \mathrm{P}<0.01$ vs $\mathrm{AC}$ (WT) (Mann-Whitney test). AC, allergic conjunctivitis; IFN- $\gamma$; interferon- $\gamma$; pERK, phosphorylated extracellular signal-regulated kinase; WT, wild type.

animals was characterised by intense influx of eosinophils into the conjunctiva compared with controls, confirming previous data from experimental models of AC. ${ }^{24} 252^{32-35}$ On the other hand, 24 hours after the final OVA challenge, the Gal- $3^{-/}$animals displayed an exacerbated local inflammatory response in relation to WT, with a greater eosinophil and mast cell influx in the palpebral conjunctiva. Furthermore, the Gal- $3^{-/}$degranulated mast cell population was larger $(\sim 50 \%)$ than the WT $(\sim 29 \%)$.

Consistent with our findings, the antimigratory effect of Gal-3 has been demonstrated in neutrophils. After preincubation with Gal-3, neutrophils reduced their migration by $20 \%-40 \%$, while their C5a, IL-8 or ATP-induced chemotaxis was inhibited by $40 \%-58 \% .^{36}$ This inhibitory effect of Gal-3 was associated with the regulation of $\mathrm{p} 38$ kinase activation and increased CD11b expression. Similarly, studies with a zymosan-induced peritonitis model detected increased neutrophil recruitment in Gal- $3^{-}$ 1. animals after 4, 72 and 96 hours. ${ }^{37}$ The results were associated with a decrease in the rate of apoptosis of Gal- $3^{-1-}$ neutrophils and their efferocytosis by macrophages.

Gal-3 has been shown to be a negative regulator of mast cell degranulation induced by IgE receptor activation (FcERI). ${ }^{38} \mathrm{In}$ this study, murine mast cells silenced for the Gal-3 gene (Gal-3 $\mathrm{KD}$ ) showed exacerbated degranulation in comparison to non-silenced cells after IgE stimulation. Gal-3 KD mast cells significantly increased mRNA production for TNF- $\alpha$, IL-6, IL-13 and CCL3 and prostaglandin D2. These findings were associated with increased tyrosine phosphorylation of various signalling factors (SYK, PLC $\gamma 1$, JNK and AKT) on Gal-3 KD mast cells, indicating that Gal-3 acts on the immediate activation of degranulation and calcium response. In addition, KD mast cells had increased migration after stimulation with IgE and prostaglandin E2 compared with control cells.

The importance of mast cells in the induction of the allergic response was demonstrated in a model of AC induced by pollen. ${ }^{39}{ }^{40}$ Mast cell-deficient mice did not present clinical signs and eosinophilia in the conjunctiva as severely as WT animals after challenge with pollen in the conjunctival sac. This effect was reversed when deficient animals were repopulated with mast cells, showing that these cells are essential in inducing the initial response to AC (clinical signs) and the recruitment of eosinophils.

In our study, the absence of endogenous Gal-3 in AC animals also caused a significant increase in eotaxin, IFN- $\gamma$, IL-4 and IL-13 levels in tear fluid as well as a local influx of $\mathrm{CD}^{+} \mathrm{T}$ cells. Reinforcing the anti-inflammatory effect of Gal-3 on AC, we showed that Gal- ${ }^{-/-}$lymphocytes displayed increased levels of nuclear and cytoplasmic pERK compared with WT cells. In fact, previous reports indicate that the activation of ERK favours the development of Th2-type immune responses. ${ }^{41}{ }^{42}$ Furthermore, studies using $\mathrm{AC}^{43}$ and asthma ${ }^{44}$ models induced by pollen and OVA, respectively, showed a significant reduction in the clinical signs of these diseases and the influx of leucocytes to tissues in IFN- $\gamma$ knockout mice compared with WT animals. In these same studies, the researchers found that the neutralisation of IFN- $\gamma$ in WT animals by treatment with a specific antibody induced similar effects to those observed for knockout animals. 
In addition, in vitro assays demonstrated that IFN- $\gamma$ is able to activate mast cells, previously sensitised with $\operatorname{IgE}$ antibodies, inducing the release of histamine, IL- 6 and IL-13 after a 1 hour or 24 hours challenge with specific antigens. ${ }^{44}$

Collectively, our study reveals the role of Gal-3 in the pathogenesis of ocular allergy, regulating the eosinophil migration, mast cell activation and production of local cytokines/chemokines and constituting a relevant therapeutic target.

Acknowledgements The authors thank Fundação de Amparo à Pesquisa do Estado de São Paulo (FAPESP_Grant 2015/09858-3).

Contributors FECA and CDG performed the experiments. All authors contributed to the sample collection and data analysis/interpretation. FECA and CDG wrote the manuscript. All authors have reviewed and approved the final version of manuscript. CDG conceived and designed the study.

Funding FECA was supported by Coordenação de Aperfeiçoamento de Pessoal de Nível Superior (CAPES).

Competing interests None declared.

Patient consent Obtained.

Ethics approval Ethical Committee of Federal University of São Paulo (CEP $n^{\circ}$ 1.151.068)

Provenance and peer review Not commissioned; externally peer reviewed.

(c) Article author(s) (or their employer(s) unless otherwise stated in the text of the article) 2018. All rights reserved. No commercial use is permitted unless otherwise expressly granted.

\section{REFERENCES}

1 Thiemann S, Baum LG. Galectins and Immune Responses-Just How Do They Do Those Things They Do? Annu Rev Immunol 2016;34:243-64.

2 Gil CD, Cooper D, Rosignoli G, et al. Inflammation-induced modulation of cellular galectin-1 and -3 expression in a model of rat peritonitis. Inflamm Res 2006;55:99-107.

3 Gil CD, La M, Perretti M, et al. Interaction of human neutrophils with endothelial cells regulates the expression of endogenous proteins annexin 1, galectin-1 and galectin-3. Cell Biol Int 2006;30:338-44.

4 Ge XN, Bahaie NS, Kang BN, et al. Allergen-induced airway remodeling is impaired in galectin-3-deficient mice. J Immunol 2010;185:1205-14.

5 Dabelic S, Novak R, Goreta SS, et al. Galectin-3 expression in response to LPS, immunomodulatory drugs and exogenously added galectin-3 in monocyte-like THP-1 cells. In Vitro Cell Dev Biol Anim 2012;48:518-27.

6 Radosavljevic G, Volarevic V, Jovanovic I, et al. The roles of Galectin-3 in autoimmunity and tumor progression. Immunol Res 2012;52:100-10.

7 Gruson D, Ko G. Galectins testing: new promises for the diagnosis and risk stratification of chronic diseases? Clin Biochem 2012;45:719-26.

8 Jeng KC, Frigeri LG, Liu FT. An endogenous lectin, galectin-3 (epsilon BP/Mac-2), potentiates IL-1 production by human monocytes. Immunol Lett 1994;42:113-6.

9 Zuberi RI, Frigeri LG, Liu FT. Activation of rat basophilic leukemia cells by epsilon BP, an IgE-binding endogenous lectin. Cell Immunol 1994;156:1-12.

10 Sano H, Hsu DK, Yu L, et al. Human galectin-3 is a novel chemoattractant for monocytes and macrophages. J Immunol 2000;165:2156-64.

11 Sano H, Hsu DK, Apgar JR, et al. Critical role of galectin-3 in phagocytosis by macrophages. J Clin Invest 2003;112:389-97.

12 Rao SP, Wang Z, Zuberi RI, et al. Galectin-3 functions as an adhesion molecule to support eosinophil rolling and adhesion under conditions of flow. J Immunol 2007; 179:7800-7

13 Ge XN, Ha SG, Liu FT, et al. Eosinophil-expressed galectin-3 regulates cell trafficking and migration. Front Pharmacol 2013;4:37.

14 Gittens BR, Bodkin JV, Nourshargh S, et al. Galectin-3: A Positive Regulator of Leukocyte Recruitment in the Inflamed Microcirculation. J Immunol 2017; 198:4458-69.

15 Saegusa J, Hsu DK, Chen HY, et al. Galectin-3 is critical for the development of the allergic inflammatory response in a mouse model of atopic dermatitis. Am J Pathol 2009;174:922-31.

16 del Pozo V, Rojo M, Rubio ML, et al. Gene therapy with galectin-3 inhibits bronchial obstruction and inflammation in antigen-challenged rats through interleukin-5 gene downregulation. Am J Respir Crit Care Med 2002;166:732-7.
17 López E, del Pozo V, Miguel T, et al. Inhibition of chronic airway inflammation and remodeling by galectin-3 gene therapy in a murine model. $J$ Immunol 2006;176:1943-50.

18 López E, Zafra MP, Sastre B, et al. Gene expression profiling in lungs of chronic asthmatic mice treated with galectin-3: downregulation of inflammatory and regulatory genes. Mediators Inflamm 2011;2011:1-9.

19 Kumar S. Vernal keratoconjunctivitis: a major review. Acta Ophthalmol 2009;87:133-47

20 Liendo VL, Vola ME, Barreiro TP, et al. Topical tacrolimus for the treatment of severe allergic keratoconjunctivitis in children. Arq Bras Oftalmol 2017:80:211-4.

21 Müller EG, Santos MSD, Freitas D, et al. Tacrolimus eye drops as monotherapy for vernal keratoconjunctivitis: a randomized controlled trial. Arq Bras Oftalmol 2017; 80:154-8.

22 Paris FS, Gonçalves ED, Barros JN, et al. Impression cytology findings in bullous keratopathy. Br J Ophthalmol 2010;94:773-6.

23 Hsu DK, Yang RY, Pan Z, et al. Targeted disruption of the galectin-3 gene results in attenuated peritoneal inflammatory responses. Am J Pathol 2000;156:1073-83.

24 Mello CB, Ramos L, Gimenes AD, et al. Immunomodulatory effects of galectin-1 on an IgE-mediated allergic conjunctivitis model. Invest Ophthalmol Vis Sci 2015;56:693-704.

25 Gimenes AD, Andrade TR, Mello CB, et al. Beneficial effect of annexin A1 in a mode of experimental allergic conjunctivitis. Exp Eye Res 2015;134:24-32.

26 Barequet IS, Platner E, Sade K, et al. Topical tacrolimus for the management of acute allergic conjunctivitis in a mouse model. Graefes Arch Clin Exp Ophthalmol 2013;251:1717-21.

27 Vichyanond P, Pacharn P, Pleyer U, et al. Vernal keratoconjunctivitis: a severe allergic eye disease with remodeling changes. Pediatr Allergy Immunol 2014;25:314-22.

28 Singh R, Joseph A, Umapathy T, et al. Impression cytology of the ocular surface. Br J Ophthalmol 2005;89:1655-9.

29 Barros JN, Almeida SR, Lowen MS, et al. Impression cytology in the evaluation of ocular surface tumors: review article. Arq Bras Oftalmol 2015;78:126-32.

30 Han JL, Ding RY, Zhao L, et al. Rosiglitazone attenuates allergic inflammation and inhibits expression of galectin-3 in a mouse model of allergic rhinitis. J Int Med Res 2008;36:830-6.

31 Zuberi RI, Hsu DK, Kalayci O, et al. Critical role for galectin-3 in airway inflammation and bronchial hyperresponsiveness in a murine model of asthma. Am J Pathol 2004; 165:2045-53.

32 Shoji J, Sakimoto T, Muromoto K, et al. Comparison of topical dexamethasone and topical FK506 treatment for the experimental allergic conjunctivitis model in BALB/C mice. Jpn J Ophthalmol 2005;49:205-10.

33 Giavina-Bianchi P, Kalil J, Rizzo LV. Development of an animal model for allergic conjunctivitis: influence of genetic factors and allergen concentration on immune response. Acta Ophthalmol 2008;86:670-5.

34 Chung SH, Nam KH, Kweon MN. Staphylococcus aureus accelerates an experimental allergic conjunctivitis by Toll-like receptor 2-dependent manner. Clin Immunol 2009;131:170-7.

35 Fukushima A, Ishida W, Ojima A, et al. Participation of CD11b and F4/80 molecules in the conjunctival eosinophilia of experimental allergic conjunctivitis. Int Arch Allergy Immunol 2010;151:129-36.

36 Baseras B, Gaida MM, Kahle N, et al. Galectin-3 inhibits the chemotaxis of human polymorphonuclear neutrophils in vitro. Immunobiology 2012;217:83-90.

37 Wright RD, Souza PR, Flak MB, et al. Galectin-3-null mice display defective neutrophil clearance during acute inflammation. J Leukoc Biol 2017;101:717-26.

38 Bambouskova M, Polakovicova I, Halova I, et al. New Regulatory Roles of Galectin-3 in High-Affinity IgE Receptor Signaling. Mol Cell Biol 2016;36:1366-82.

39 Miyazaki D, Tominaga T, Yakura K, et al. Conjunctival mast cell as a mediator of eosinophilic response in ocular allergy. Mol Vis 2008;14:1525-32.

40 Fukuda K, Ohbayashi M, Morohoshi K, et al. Critical role of IgE-dependent mast cell activation in a murine model of allergic conjunctivitis. J Allergy Clin Immunol 2009;124:827-33.

41 Dillon S, Agrawal A, Van Dyke T, et al. A Toll-like receptor 2 ligand stimulates Th2 responses in vivo, via induction of extracellular signal-regulated kinase mitogen-activated protein kinase and c-Fos in dendritic cells. J Immunol 2004; 172:4733-43.

42 Agrawal A, Dillon S, Denning TL, et al. ERK1-/- mice exhibit Th1 cell polarization and increased susceptibility to experimental autoimmune encephalomyelitis. J Immunol 2006; 176:5788-96.

43 Stern ME, Siemasko KF, Niederkorn JY. The Th1/Th2 paradigm in ocular allergy. Curr Opin Allergy Clin Immunol 2005;5:446-50.

44 Yu M, Eckart MR, Morgan AA, et al. Identification of an IFN-?]/mast cell axis in a mouse model of chronic asthma. J Clin Invest 2011;121:3133-43. 